\title{
SURGERY IN EMPHYSEMA AND ASTHMA
}

\author{
By L. L. Bromley, M.Chir., F.R.C.S. \\ Surgeon in Charge of the Thoracic Department, St. Mary's Hospital, London
}

Emphysema and asthma are two chronic lung diseases which are often together associated with chronic bronchitis. The interrelationship of these three conditions is complex and poorly understood, but there is a great surge of interest in this field which presents a vast clinical problem in an ageing population. Asthma is due to transient narrowing of large air tubes caused by acute mucosal swelling with or without associated spasm of the bronchial musculature and is initiated by allergic or psychogenic stimuli. The essential lesion in chronic bronchitis seems likely to be a hypersecretion of mucus from large and small air tubes, there is interference with normal bronchial ciliary action and infection develops as a secondary manifestation. Chronic bronchitis and asthma are diseases of bronchi and in both there is, at times, acute or chronic bronchial obstruction which is incomplete. Emphysema, on the other hand, is a disease of distal lung tissue; its cause is not known, but it seems certain that it is very intimately related to partial bronchial obstruction.

\section{Emphysema}

Surgical treatment will be considered in relation to various types of emphysema as they present clinically:

I. Obstructive emphysema.

2. Acute emphysema in infancy.

3. Pure hypertrophic emphysema.

4. Bullous emphysema.

5. Pneumothorax complicating emphysema.

\section{Obstructive Emphysema}

The commonest cause of obstruction is an inhaled foreign body. Fig. I. shows emphysema affecting the middle and lower lobes of the right lung due to an inhaled peanut which has produced valvular obstruction in the descending (intermediate) bronchus. This acute process, where there is trapping of air distal to the obstruction, is quite reversible. It is seen initially following inhalation of the foreign body, but soon the picture can change to that of lobar collapse as inflammatory oedema at the site of lodgement now adds to the obstruction and makes it complete. Removal

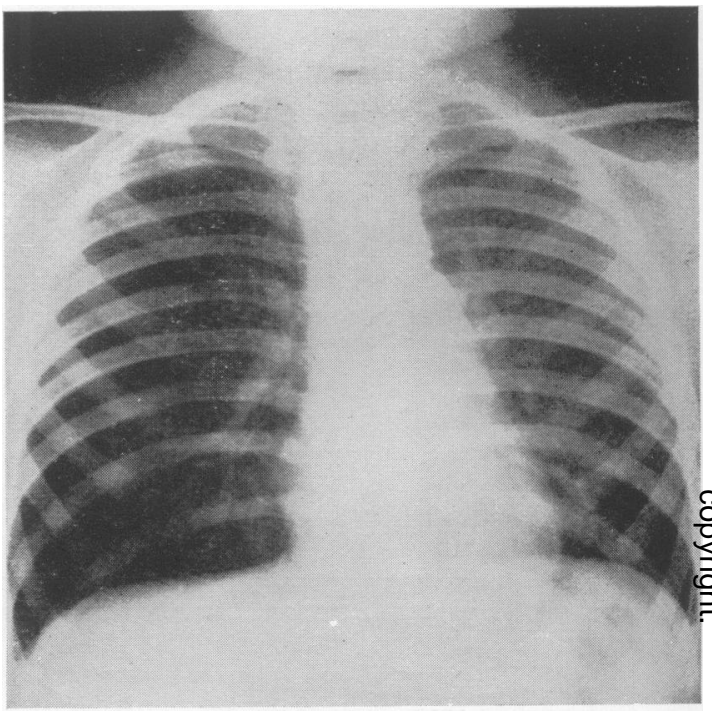

FIG. 1.-Obstructive emphysema of the middle and lower lobes of the right lung due to an inhaled foreign body.

of the foreign body through a bronchoscope at the stage of obstructive emphysema is the best insurance against further trouble, for if collapse ensues, and distal infection in the lung develops, irreversible bronchiectatic changes may occur. The discovery of zonal emphysema in a child, with or without a history of inhalation of a foreign body, makes bronchoscopy a most necessary and urgent examination.

In older children and in adults localized emphysema of a lobe or of a whole lung may be encountered, and here the mechanism can sometimes be shown to be a valvular obstruction produced by a redundant fold of mucous membrane or by an abnormality of the bronchial cartilages, so that an extremely lax bronchial wall is present. Bronchoscopy and bronchography may be of help in assessing this condition. Surgery is only necessary where there are symptoms, such as dyspnoea or those of recurring pulmonary infections, and if there is obvious displacement and 


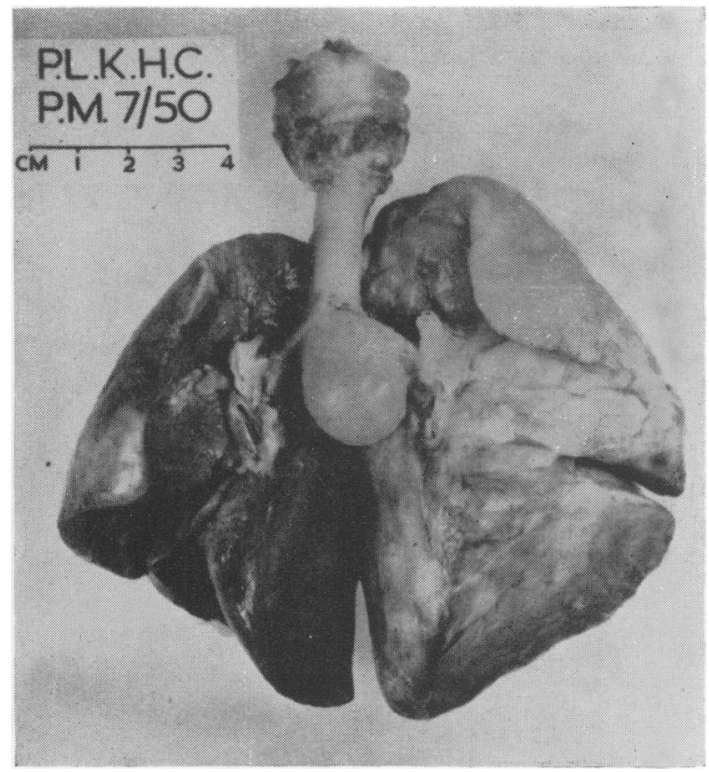

FIG. 2.-Emphysema of the left lung due to a congenital bronchial cyst.

compression of normal lung. Excision of the involved lobe is indicated; very rarely it may be found possible to provide support to a bronchus which has a deficiency of cartilage by the intramural placement of wire sutures (Cleland, 1956).

\section{Acute Emphysema in Infants}

Infants can develop gross emphysema and this may appear within a few days of birth and progress with very great rapidity. It causes tachypnoea, cyanosis during feeds, a tympanitic percussion note and bulging of the chest on the affected side. The condition would appear to be acute obstructive emphysema, but the actual cause of the obstruction is seldom apparent: in most cases there is a giant air cyst within a lobe which expands and compresses normal lung; in others one lobe is grossly enlarged due to generalized distention of alveoli. Fig. 2 shows acute emphysema in an infant where there is an obvious cause; a congenital bronchial cyst (from misplaced foregut tissues) lies just below the carina and is pressing on the left main bronchus thus producing unilateral obstructive emphysema. In early infancy there may be some difficulty in distinguishing emphysema from a large diaphragmatic hernia where, however, intestinal symptoms are usually present and a bowel pattern may be evident on the chest radiograph. Thoracotomy is indicated for infants presenting with progressive dyspnoea due to emphysema, as they are unlikely to survive without operation. The early part of the procedure may be hazardous, but as soon as the infant's chest is open ventilatory conditions improve and the emphysematous lung can be excised without haste or undue difficulty; a lobectomy is the usual required procedure. The results of surgery are excellent; the remaining lobes expand readily to fill the space previously occupied by the emphysematous lung.

\section{Pure Hypertrophic Emphysema}

Surgical treatment in this very common type of emphysema has little to offer the patient. The disease is diffuse, generalized and bilateral. Pneumoperitoneum has had its advocates, but lung function studies performed before and after establishing the pneumoperitoneum have shown that there is no lasting increase in the maximum breathing capacity nor in exercise tolerance (Mann and Murphy, 1954). Attempts have been made to treat emphysema by operations on the autonomic nervous system with the proposed rationale that emphysema, if not actually due to incomplete bronchial obstruction, is made worse by it, and that if the air tubes could be widened air flow in the lungs would be unhindered and air trapping would be avoided. In order to remove all bronchoconstrictor fibres, both parasympathetic and sympathetic fibres must be divided, but the results of operations for pure hypertrophic emphysema have been disappointing (Abbott, I953). Crenshaw and Rowles (I952) recommended excision of the parietal pleura combined with neurectomy for emphysema. They considered that this might promote the growth of vascular adhesions between the emphysematous lung and the chest wall and increase the blood flow to lung undergoing progressive degeneration due to a poor systemic blood supply. It is difficult to assess the possible benefits that this procedure may afford, as it has been combined with neurectomy and its isolated use has not been reported.

It is hard to foresee how surgery may develop so as to help these patients unless some prophy. lactic procedure emerges. It seems likely that this diffuse form of the disease will remain a medical problem.

\section{Bullous Emphysema}

It is in this type of emphysema that surgery has a great deal to offer, particularly in improving exercise tolerance. In the lungs of these patients there are three macroscopical features, blebs, bullae and hypertrophic emphysema. Blebs are superficial air cysts, usually not larger than $3 \mathrm{~cm}$. in diameter, and are immediately subpleural, appearing to be due to the rupture of superficial alveoli with the production of blisters on the surface of the lung. Bullae, also known as pneumato- 


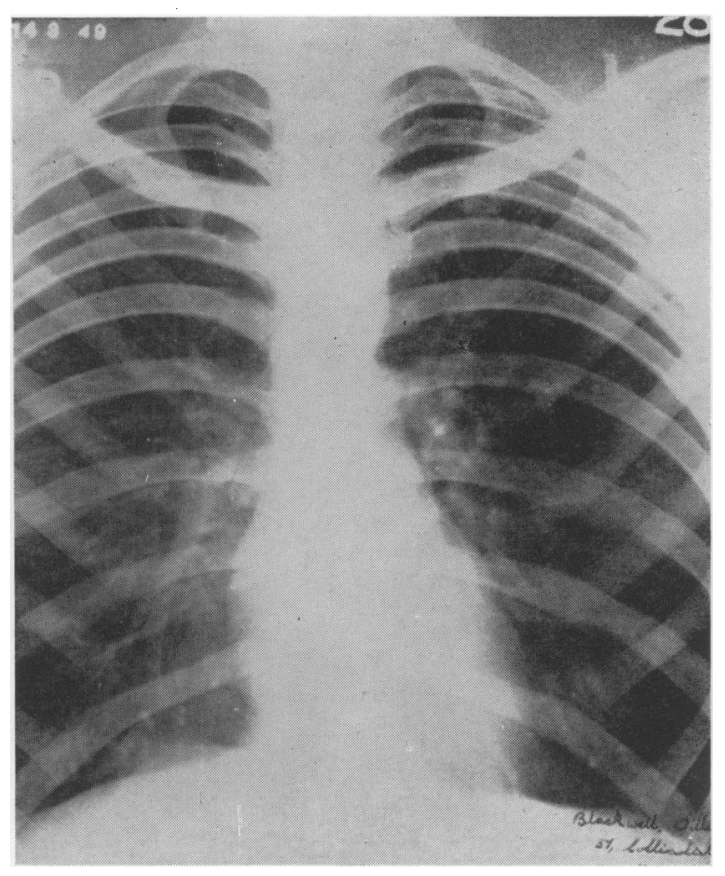

FIG. 3.-X-ray chest of an asthmatic man aged $2 \mathrm{r}$.

coeles, are within the lung substance and are primarily due to intrapulmonary rupture of alveoli or terminal bronchioles with progressive distension of the lung parenchyma. They are multiple, one or two are larger than others and by their bulk they ultimately compress surrounding lung. The severity of the third feature, hypertrophic emphysema, varies greatly; it is most often seen at the fringes of the lobes. It is important to realize that bullae and blebs take no part in respiration, as there is a negligible gas exchange through the few small bronchiolar or alveolar connections with their interior. Although the pressure within the bullae is only a few centimetres of water above atmospheric, it is, nevertheless, high enough to compress surrounding lung and the bullae act as intruders on the space required by normal alveoli.

Patients with bullous emphysema may be young, but most are over 50. They give a history of progressive diminution of exercise tolerance and this may reach the stage of dyspnoea and cyanosis on the slightest exertion. Many of the patients give a history of asthma and of mild recurrent pulmonary infections. Fig. 3 is the radiograph of a man aged $2 \mathrm{I}$ who was subject to asthmatic attacks; it appears normal. Fig. 4 is of the same man five years later and it shows the replacement of the upper parts of each lung field by giant bullae, with now gross limitation of exercise tolerance.

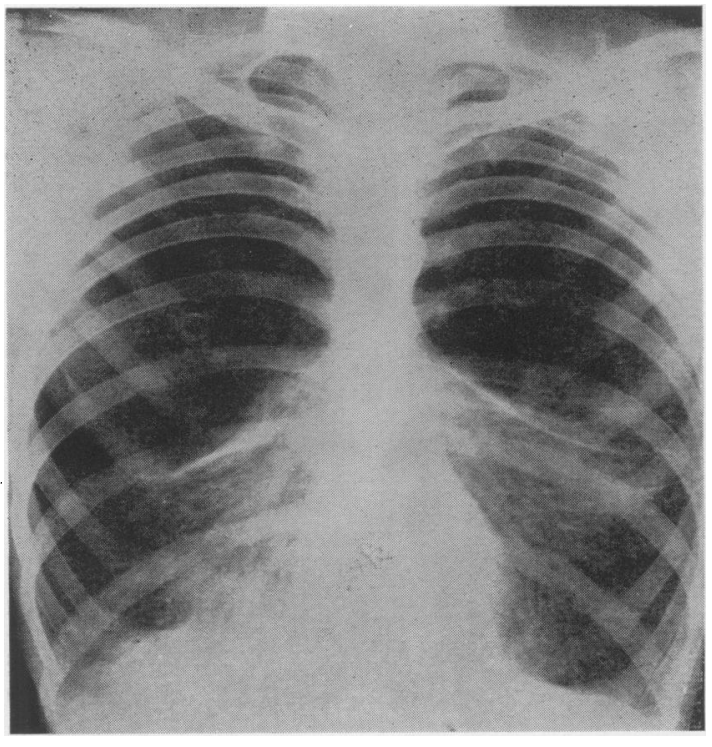

FIG. 4.- Same man as in Fig. 3, now aged 26, showing giant bullae on each side.

The investigation of such patients is important and should include bronchoscopy to exclude are $\vec{e}$ endobronchial abnormality and to assess the degree of of any bronchial distortion that may be presento Fluoroscopy and bronchography are helpful and pulmonary function tests are undoubtedly of value and if facilities exist they should be performed on all patients with bullous emphysema. Function tests will provide some objective evidence of the type and degree of impairment of lung function and the results of surgery can be to some extent quantitated. However, although very desirable, they are not absolutely necessary in those cases where the bullae are reasonably localized and the patient is clearly short of breath as a result of them.

\section{Surgical Treatment}

In all cases with symptoms treatment is initially on medical lines. Tobacco should be renounced; any nasal, oral or pulmonary infection should be appropriately treated; anti-spasmodics should be given and breathing exercises taught. It has been shown that, whilst breathing exercises do $\widetilde{\sigma}$ not actually increase lung function, they do result $N$ in improved diaphragmatic movement, and this, if operation is undertaken, is an important and valuable acquisition. Operation is recommended if disabling shortness of breath is still present after a period of full medical treatment. Thoracotomy $\stackrel{\infty}{\rightarrow}$ is performed under general anaesthesia and immediately the parietal pleura is opened the largest bulla will herniate through the incision.

Fig. 5 shows a large bullous cyst of the left 


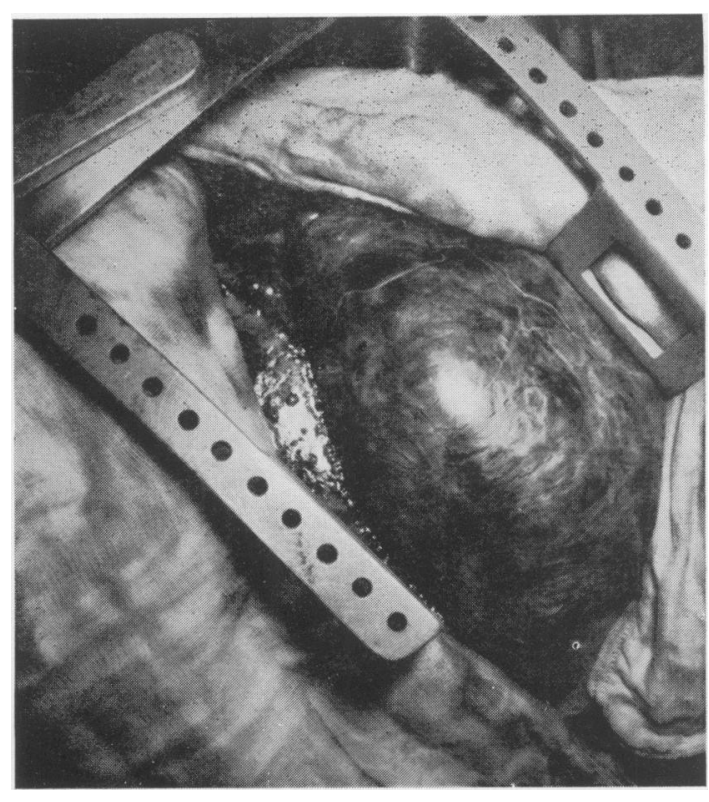

Fig. 5.-Bullous cyst of left upper lobe at operation.

upper lobe; the ribs have just been spread apart. In order to appreciate the situation fully a small nick is made in the wall of the presenting bulla, which causes its partial collapse and allows room for thorough inspection of the whole lung. As already stated, it is usual to find blebs and zones of hypertrophic emphysema besides other smaller bullae. The possibilities that are now open to the surgeon are pneumonectomy, lobectomy, segmental resection or simple plication obliteration of the cyst. As a general principle, the surgeon's attitude should be to preserve as much normal lung tissue as possible and with this aim in view the most satisfactory form of surgery is cyst plication. The bulla is opened more widely and any bronchial communications that can be identified are closed by fine silk stitches; it is then obliterated by a combination of suture of its walls and the invagination and suture of its thin presenting outer shell. Smaller cysts and blebs can be similarly managed. Lobectomy or segmental resection is justifiable only with very localized disease. The lung should be made as airtight as possible before closure of the chest and it is important that the anaesthetist is not encouraged to over-inflate the lung in order to make it fill the hemithorax immediately. It will not do so at once, and there is great danger of seeing new blebs, and even bullae, appear from new intrapulmonary air dissections as a result of over-enthusiastic hyperinflation. Autonomic neurectomy and removal of the parietal pleura has already been mentioned and can now be carried out. Although there is no certain evidence that these procedures will help to prevent the development of further emphysema, and it would seem very difficult to see how such evidence is to be obtained in man, there is possibly some justification for carrying them out where there is asthma in association with bullous emphysema. Opinions differ, but it is considered that an open mind should be kept, for emphysema is not necessarily a single disease: it is possibly due to a combination of influences and surgical treatment might logically be directed at the outside connections of the lung as well as at the lung itself. Neurectomy may be empirical, but no evidence has yet been presented showing that harm can be done, although inevitably it adds time to the operation. Apical and basal drainage tubes are inserted and moderate suction applied to them. The lung will take some time to expand sufficiently to fill the hemithorax; the tubes are removed when air leaks stop; any residual pneumothorax is repeatedly aspirated and when small allowed to absorb slowly.

The operative mortality depends on the type of patient accepted, but generally the surgery is well tolerated and there is immediate improvement in the patient's breathing and capabilities. Surgery can be undertaken even in the very breathless individual, but such patients require especially careful preparation. For the critically disabled patient with dyspnoea at rest and cyanosis there is sometimes a place for Monaldi type suction drainage (Head and Avery, 1949), where a twostage procedure is usually required. At the first, pleural adhesions are locally produced by leaving a gauze pack against the outer aspect of the parietal pleura, having resected a small segment of rib overlying the bulla; two weeks later the pack is removed and a catheter is introduced into the bulla, there now being no danger of producing a pneumothorax. Suction is applied and in a small bulla it may be possible, over the next ro days, to suck the walls together and, with their adherence, obliterate the air space. In the larger bullae, however, a limited reduction in size is achieved, but this may put the patient in a better position to undergo thoracotomy.

\section{Pneumothorax Complicating Emphysema}

Spontaneous pneumothorax may be a complication of emphysema, of the hypertrophic or of the bullous type and often there is a tension pneumothorax. Recurrent episodes are common. A patient with emphysema who develops a tension pneumothorax is in a critical position, as he has little respiratory reserve, thus early diagnosis and treatment are important. For the acute episode aspiration of air from the pleural cavity may be urgently 
necessary and if, after an initial aspiration, air reaccumulates, the needle should be left in the chest and connected to an under-water seal, but if this channel of exit for air is not large enough an intercostal tube must be inserted and similarly connected to an under-water seal. After the acute episode is over the condition must be critically assessed, particularly if this has been the latest of a succession of episodes or if pneumothoraces have occurred on each side. If large bullae are present in the underlying lung, thoracotomy is advised and plication of the bullae carried out. Pleural adhesions will follow the thoracotomy and a further pneumothorax cannot occur. Where the bullae are small or pure hypertrophic emphysema is present and the pneumothorax persists thoracoscopy is done, the lung is painted with ro per cent. silver nitrate and $0.5 \mathrm{ml}$. of the same solution sprayed against the parietal pleura of the paravertebral gutter and a tube connected to a water seal left temporarily in the chest. An effusion develops which must later be aspirated as required; adhesion of the two layers of the pleura results. Where the pneumothorax has completely absorbed but there have been several episodes of leak from the lung, air should be introduced into the pleural cavity and into this induced pneumothorax $1.5 \mathrm{ml}$. of silver nitrate is injected; aspiration of the effusion that forms is carried out.

This approach towards a pneumothorax complicating emphysema may appear aggressive, but patients are in constant and considerable danger if a laisser-faire attitude is adopted and treatment confined only to the initial management of the tension pneumothorax; if further attacks occur, treatment may not be available to the patient in time.

\section{Asthma}

The only surgical treatment of asthma that need be considered is autonomic surgery and presentday opinion is that it has little effect on the condition. It would appear, however, to have a more rational basis than autonomic denervation of the lung for emphysema, as asthmatic attacks can be $\stackrel{\vec{\rho}}{\stackrel{5}{+}}$ initiated by psychogenic stimuli, whilst vagal stimulation in the experimental animal, and in 흠 man, has been shown to produce intense broncho- $\frac{\bar{T}}{\partial}$ spasm. Ideally autonomic surgery would be best $\stackrel{\mathbb{D}}{\stackrel{\alpha}{\alpha}}$ applied to the asthmatic with no allergic component to his condition, but such sufferers are rare. In order to divide all bronchoconstrictor fibres to the $\vec{\circ}$ lungs, both sides of the chest must be operated $\overrightarrow{\vec{\omega}}$ upon, and it is necessary to divide all vagal $\stackrel{\omega}{\sigma}$ branches to the anterior and posterior pulmonary 8 plexuses, to perform an upper dorsal sympathec- 3 . tomy down as far as the fifth ganglion, to circum- w cize the sheaths of the pulmonary vessels and to $\vec{\omega}$ divide the pulmonary ligament. There have been some encouraging reports in the literature, but $\vec{A}$ late follow-up on patients who have undergone 0 autonomic nerve surgery has shown a high in- $\stackrel{?}{-}$ cidence of return of asthma. It would seem proper $\vec{c}$ to consider surgical treatment only where all other forms of treatment have failed in a patient with intractable and severe asthma, where an allergic $₹$ element is excluded and where there is a favourabl $\overrightarrow{0}$ response to a ganglion-blocking agent, such o hexamethonium. There is yet no recognized plae for neurectomy as a standard approach to the management of the asthmatic.

\section{BIBLIOGRAPHY}

ABBO'I"I, O. A., HOPKINS, W. A., VAN FLEIT, W. E., and ROBINSON, J. S. (1953), Ibid., 8, I 16.

('LELAND, W. P. (1956), personal communication.

CRENSHAW, G. L., and ROWLES, D. F. (1952), f. thorat. Surg., 24, 398.

HEAD, J. R., and AVERY, E. E. (1949), Ibid., 18, 76 r.

MANN, B., and MURPHY, E. A. (r954), Thorax, 9, 87.

\section{RUTHIN CASTLE, NORTH WALES}

A Clinic for the diagnosis and treatment of Internal Diseases (except Mental or Infectious Diseases). The Clinic is provided with a staff of doctors, technicians and nurses.

The surroundings are beautiful. The climate is mild. There is central heating throughout. The annual rainfall is $\mathbf{3 0 . 5}$ inches, that is less than the average for England.

The Fees are inclusive and vary according to the room occupied.

For particulars apply to THE SECRETARY, Ruthin Castle, North Wales. 